\title{
On-Eye Measurement of Optical Performance of Rigid Gas Permeable Contact Lenses Based on Ocular and Corneal Aberrometry
}

\author{
CARLOS DORRONSORO, MSc, SERGIO BARBERO, MSc, LOURDES LLORENTE, OD, \\ and SUSANA MARCOS, PhD \\ Instituto de Óptica "Daza de Valdés", Consejo Superior de Investigaciones Cientificas, Madrid, Spain
}

\begin{abstract}
Purpose. Our aim was to obtain a complete description of the interactions of rigid gas permeable (RGP) contact lenses with the optics of normal eyes. Methods. We measured total and anterior-surface aberrations in four subjects, who were all long-term RGP contact lens wearers. The anterior-surface wave aberration was obtained from videokeratographic elevation maps, and ocular wave aberration was measured with a laser ray-tracing technique. Measurements were performed with and without their own spherical contact lenses. Results. With this methodology, we evaluated the optical performance with RGP lenses compared with the natural optics. We estimated the contribution of the anterior surface of the contact lens, the internal ocular optics, flexure, and the tear lens aberrations to the optical performance of eyes wearing RGP contact lenses. We found that in three of four subjects, the contact lens significantly improved the natural optics of the eye. For the subject with higher dominance of corneal aberrations, root mean square (second-order and higher) decreased from $1.36 \mu \mathrm{m}$ to $0.46 \mu \mathrm{m}$. Third- and higher-order aberrations decreased from $0.77 \mu \mathrm{m}$ to $0.39 \mu \mathrm{m}$. The internal optics and lens flexure imposed limits on aberration compensation. Spherical RGP contact lenses did not produce spherical aberration potentially due to a compensatory role of the tear lens. Conclusions. Aberration measurements are useful to understand the fitting of contact lenses and the interaction with tear, cornea, and internal optics of the eye. Aberrometry can help to choose the best standard RGP lens parameters to improve the optics of individual eyes. (Optom Vis Sci 2003;80:115-125)
\end{abstract}

Key Words: contact lens, rigid gas permeable, corneal aberration, corneal topography, videokeratography, ray tracing, aberration correction, spherical aberration, flexure, contact lens fitting, tear lens

$\mathrm{T}$ he measurement of ocular optical aberrations has proved to be useful to understand the optical properties and imageforming capabilities of the human eye. Aberrometers have started reaching the clinical environment. These new tools have already proved to be informative for studying changes in optical quality after surgical procedures (such as corneal refractive surgery, ${ }^{1,2}$ keratoplasty, ${ }^{3}$ or intraocular lens implantation after cataract surgery ${ }^{4,5}$ ), biological processes (such as aging ${ }^{6,7}$ or accommodation $^{8}$ ), or pathological conditions (i.e., keratoconus 9 or myopia $^{10,11}$ ). Wavefront sensors or aberrometers measure the wave aberrations of the entire ocular system, typically in less than a few seconds. ${ }^{12,13}$ Current videokeratographers provide a rapid and accurate description of the corneal shape. ${ }^{14}$ Several authors have used corneal elevation data from commercial corneal topography systems to estimate corneal wave aberrations. ${ }^{9}{ }^{15-18}$ The corneal wave aberration can then be subtracted from the whole-eye wave aberration to obtain the aberrations of the internal optics. Recently, several methods have been proposed and demonstrated to correct high-order aberrations of the human eye. These methods range from dynamic corrections (such as adaptive optics ${ }^{19,20}$ ) to static corrections (i.e., in the form of custom phase plates, ${ }^{21}$ custom contact lenses ${ }^{22}$ or customized ablations ${ }^{23,24}$ ).

In this paper, we will show the use of ocular aberrometry and corneal topography to assess optical performance of rigid gas permeable contact lenses (RGP CL) and the potential of this type of lens to significantly reduce ocular aberrations (not only defocus and astigmatism, but also high-order aberrations). The combination of total and anterior-surface aberrations measurements in the same subjects with their natural optics and RGP lens will allow us to assess aspects related to contact lens fitting and the influence of the tear film.

It is widely accepted in the clinical practice that RGP CL's 
provide the best ophthalmic correction, at least from a purely optical viewpoint. ${ }^{25}$ RGP CL's are expected to mask the anterior corneal surface with a perfectly regular surface and fill in with tear all the corneal irregularities. The refractive index similarity between the tear film and the anterior corneal surface reduces the impact of corneal aberrations. ${ }^{26}$ However, a direct comparison of the optical changes produced by RGP CL's on the anterior surface of the cornea and total optical system has not previously been reported.

The better visual response of RGP CL's, compared with soft CL's (which would produce the same magnification) or spectacles, is well documented in the optometry literature. ${ }^{25,26}$ Most of these studies are based on psychophysical measurements of visual performance and conclude that RGP CL's provide higher visual acuity and contrast sensitivity. Several studies perform computer simulations to understand the optical performance of the contact lens. ${ }^{27}$ Using computer modeling, they study the interaction of the lens with a model corneal surface and the optical contribution of the tear lens between the cornea and contact lens. Validating those simulations is difficult because they tend to simplify the problem: they do not take into account corneal irregularities, contact lens decentration and flexing, and the influence of internal optics. Other studies ${ }^{28}$ have measured the topography of the CL on the eye to study flexure on-eye, but the analysis is based on corneal elevation data rather than on corneal wave aberrations. To our knowledge, only Hong et al. ${ }^{29}$ have measured aberrations in subjects wearing RGP CL's, finding that in three of four subjects, RGP CL's provided lower aberrations than soft CL's and spectacle lenses.
In this paper, we measured total aberrations and anterior-surface aberrations in four young, healthy subjects who were long-term RGP CL wearers. We measured aberrations with and without the CL. The combination of these four types of measurements allows a complete description of the interactions of the CL with the subject's natural optics and a study of the optical implications of the RGP CL fitting. In this paper we show (1) the capability of RGP CL's to greatly reduce ocular aberrations beyond defocus, particularly in optically degraded eyes; (2) flexure effects of the RGP CL's on anterior-surface and total aberrations; and (3) the contribution of the tear lens to ocular aberrations.

\section{METHODS Subjects}

Four volunteers (two males and two females) participated in the study. RGP CL's were not fit for this particular study, but rather the subjects were selected because they were long-term, satisfied RGP CL wearers. Subjects wore their own CL's, which were all RGP with anterior spherical surfaces. Subjects were aged 18 to 33 years and had spherical refractions from -4.50 to $-8.00 \mathrm{D}$. Individual autorefractometer refractions, ages, axial lengths, anterior chamber depths, and corneal curvatures are reported in Table 1. Parameters of each CL provided by the manufacturers are also included Table 1. Apart from their ametropia, all eyes were normal, and best-corrected visual acuity was 1.00 or better. Only one

\section{TABLE 1.}

Individual ages, autorefractor refractions, axial lengths, anterior chamber depths, corneal curvatures and contact lens parameters provided by the manufacturer.

\begin{tabular}{|c|c|c|c|c|}
\hline & \multicolumn{4}{|c|}{ Subject } \\
\hline & S1 & $\mathrm{S} 2$ & $\mathrm{~S} 3$ & S4 \\
\hline Eye & OD & OD & OS & OD \\
\hline Age (yr) & 27 & 33 & 18 & 24 \\
\hline Refraction (D) & $-8.00-2.00 \times 8$ & $-4.50-1.25 \times 92$ & $-8.00-0.75 \times 168$ & $-6.75-0.50 \times 159$ \\
\hline Axial length (mm) & 25.25 & 26.14 & 26.78 & 27.02 \\
\hline Anterior chamber depth (mm) & 3.75 & 3.83 & 3.67 & 4.38 \\
\hline Corneal radius $(\mathrm{mm})$ & 7.62 & 8.21 & 8.19 & 8.02 \\
\hline Corneal asphericity & 0.15 & 0.1 & 0.18 & 0.12 \\
\hline Contact lens type & Permiflex & Permiflex Aire & Conflex Air 100 UV & Boston E.S. \\
\hline Manufacturer & Eurolent & Eurolent & Zeiss & Bausch \& Lomb \\
\hline $\begin{array}{l}\text { Front optical zone } \\
\text { radius_-videokeratoscope (mm) }\end{array}$ & 8.41 & 9.22 & 9.62 & 9.05 \\
\hline Back optical zone radius (mm) & 7.70 & 8.25 & 7.90 & 7.95 \\
\hline Front surface asphericity_nominal & 0 & 0 & 0 & 0 \\
\hline Back surface asphericity_nominal & 0 & 0 & -0.16 & 0 \\
\hline Front optical zone diameter (mm) & - & - & 8.5 & 8.5 \\
\hline Back optical zone diameter (mm) & 8.5 & 8.1 & - & 8.4 \\
\hline Back vertex power (D) & -3.50 & -3.25 & -7.00 & -5.00 \\
\hline Central thickness (mm) & 0.18 & 0.18 & 0.15 & 0.18 \\
\hline Material & $\mathrm{PMMA}+\mathrm{CAB}$ & $\begin{array}{c}\text { Silicone } \\
\text { Fluorcarbonate }\end{array}$ & $\begin{array}{l}\text { Fluor silicone } \\
\text { metacrilate }\end{array}$ & Enflufocon A \\
\hline $\mathrm{n}$ & 1.469 & 1.467 & 1.467 & 1.443 \\
\hline Lens center $(\mathrm{x}, \mathrm{y})(\mathrm{mm})^{a}$ & $(1.1,0)$ & $(-1.66,1.27)$ & $(-1.91,-0.25)$ & $(1.37,0.47)$ \\
\hline
\end{tabular}

${ }^{a}$ Relative to pupil center. Positive horizontal coordinates stand for nasal in right eyes and temporal in left eyes. Negative horizontal coordinates stand for temporal in right eyes and nasal in left eyes. Positive vertical coordinates stand for superior, and negative stand for inferior. 
eye was tested per subject, the right eye for subjects $S 1, S 2$, and $S 4$ and the left eye for subject S3. RGP lens stabilization and repositioning after blinking was checked by pupil video monitoring (with respect to the pupil center). Although there were no inter-eye differences in the rest of the subjects, in subject $S 3$, centration was significantly better for the left than for the right eye, and therefore the left eye was chosen for measurements. Table 1 reports the coordinates of the center of the CL (in its stable position) relative to the pupil center.

All subjects had an eye examination before participating in the experiment. All subjects were informed about the nature of the study and signed an informed consent form as per the tenets of the Declaration of Helsinki. The protocols and consent forms were approved by institutional review boards.

\section{General Experimental Procedure}

All measurements were conducted in the same experimental session, which lasted about an hour. Initial routine measurements included slit lamp examination, autorefraction (Automatic Refractor Model 597, Humphrey-Zeiss), and axial length and anterior chamber depth by optical biometry (IOLmaster, HumphreyZeiss). These measurements, as well as videokeratography (Atlas Mastervue Corneal Topography System Model 990, HumphreyZeiss), were obtained without the CL. A second videokeratography was obtained with the subject wearing his/her CL. Videokeratographic images were taken when the CL had reached a stable position after blinking. Images distorted by tear fluid irregularities (more frequent when the eye was wearing the CL) were rejected. Pupils were dilated by means of one drop of tropicamide $1 \%$ before laser ray-tracing measurements of ocular aberrations. The first set of measurements was taken without the CL, and the last set of measurements was taken with the CL.

\section{Anterior-Surface Aberration Measurements}

Anterior-surface aberrations were estimated from elevation maps that were obtained with the videokeratographer and custom algorithms. Without the CL, these maps represent the corneal elevation. With the CL, they represent the elevation of the anterior surface of the CL. The experimental procedure has been described in detail in earlier studies. ${ }^{1,9,17}$ In brief, the elevation raw data are fitted by a seventh-order Zernike polynomial expansion and evaluated in a regular $x-y$ sampling. This surface is introduced in an optical design program, Zemax V.9 (Focus Software, Tucson, AZ). Spot diagrams are generated by virtual ray tracing through the corneal surface, setting the index of refraction to that of the aqueous humor (1.3315), and the wavelength to that used in the laser ray-tracing (LRT) measurement (see below). Wave aberrations were estimated from the sets of deviations by modal fitting to a Zernike polynomial expansion. Realigning algorithms ${ }^{9}$ were used to ensure registration of total and corneal wave aberration maps. Mean Zernike coefficient variability is $0.015 \mu \mathrm{m}$ (averaged across terms).

\section{Total Aberration Measurements}

Setup and Procedures. Total aberrations were measured (with and without the RGP CL) using LRT. A detailed description of this method can be found elsewhere. ${ }^{12,30,31}$ A set of 37 parallel laser pencils sample the eye's pupil by means of a scanning system (using hexagonal sampling and random order). Aerial images of the retinal spots corresponding to different entry pupils are collected sequentially onto a cooled CCD camera. Collection of 37 images typically takes $<4 \mathrm{~s}$. The centroids of the set of aerial images are computed. The deviations of the centroids from the principal ray are proportional to the derivatives of the wave aberrations. The wave aberration is obtained by fitting the derivatives to a Zernike polynomial expansion (up to the seventh order) using a least mean squares procedure.

All measurements were made foveally. A red laser spot colinear to the optical axis of the instrument and entering the eye through the center of the pupil was used for fixation. A CCD camera centered at the optical axis of the instrument was used to monitor and center the pupil and also to monitor possible shifts of the RGP lens. Subjects were stabilized by means of head rest and dental impression attached to a three-dimensional positioning system.

A near infrared laser diode $(786 \mathrm{~nm})$ was used as a light source. Measurements on one subject (see below) were performed with both $543-\mathrm{nm}$ and 786-nm illumination. Recent experiments ${ }^{32}$ have shown that third-and higher-order aberrations are equivalent in these two wavelengths. Light exposure was at least two orders of magnitude below safety limits.

Typical pupil diameters for LRT measurements in previous studies were $6.5 \mathrm{~mm}$ (with a sample step of $1 \mathrm{~mm}$ ). In this study we reduced maximum pupil size $(6 \mathrm{~mm}$ for subject $S 1,5.5 \mathrm{~mm}$ for subjects $\mathrm{S} 3$ and $\mathrm{S} 4$, and $5 \mathrm{~mm}$ for subject $\mathrm{S} 2$ ). We found that for larger pupil diameters, several images (corresponding to the most eccentric entry pupils) were affected by diffraction at the edge of the CL. The sampling step was varied such that in all cases, the pupil was sampled by 37 rays. For comparison purposes, all data were recomputed for 5 -mm pupils.

The largest contribution to the displacement of retinal aerial images in measurements without CL's was caused by spherical errors. For the pupil diameters used, all the aerial images fit within the CCD chip, except for one subject (S3), for whom spherical errors moved the aerial image outside the CCD. For this subject, we compensated for the refractive error with a trial lens $(-7 D)$ in measurements performed without the CL. For one subject (S4), we conducted measurements with and without trial lens to assess any possible contribution of the trial lens correction (see below).

Control and Trial Experiments: Pupil Monitoring. Similar to videokeratographic image capture with CL's, some training was required to optimize image capture with CL's in LRT and to ensure that measurements were taken with the lens in its stable position. Initial measurements were performed in one subject wearing RGP CL (S1) using green light $(543 \mathrm{~nm})$. The pupil was illuminated by infrared $(780 \mathrm{~nm})$ light using a ring optical fiber illuminator. A filter $(543 \mathrm{~nm})$ was placed in front of the CCD camera that captured the aerial images to eliminate spurious light from the pupil illumination. A frame grabber captured the video signal from the pupil monitoring camera, whereas the test beam scanned the pupil, and the second camera captured the aerial images. Pupil images also show the position of the CL and the first Purkinje images of the sampling beam (actually the reflection comes from the CL rather than from the anterior corneal surface) as it moves across the pupil. With this configuration, we were able 
to assess the exact entry pupil location for each captured aerial image. In all cases, aerial images showing a diffraction pattern and elongated in a direction perpendicular to the CL edge corresponded to rays that hit the edge of the optical zone of the CL. We also were able to assess CL motion dynamics in all subjects by pupil monitoring. All of the CL's moved downward significantly when the subject raised his/her upper lid more than normal. After some feedback, the subject was able to maintain good CL stability.

We performed measurements on subject S1 using both visible (543 nm) and infrared (786 nm) light. Except for exceptional runs for which the CL was clearly displaced (as assessed by the pupil video image during the measurements in green), results in both wavelengths were within the measurement variability (average standard deviation across Zernike coefficients $<0.1 \mu \mathrm{m}$ ). For the sake of the subjects' comfort, only infrared light was used for the rest of the subjects. Dynamics of the CL were assessed with the described system before the measurement, and when stability was achieved, pupil illumination was turned off during aerial image capture in infrared light.

Control and Trial Experiments: Effect of Trial Lenses. Trial lenses, or in general any correction system (i.e., Badal optometer) that changes ray convergence to optimize retinal focus, may have an effect on the measured spherical aberration. We measured one subject (S4) with his uncorrected eye and with a trial lens $(-5$ D) in front of the eye. The converging effect of the lens introduces a scaling in the sampling pattern, which was corrected by the software controlling the scanner. We could not find significant differences in the aberrations measured with and without the trial lens.

\section{Data Handling and Selection}

Special care was taken in the processing of data from eyes wearing CL's because they were subject to problems not present in the natural eyes (lens movement or partial pupil covering by the eyelid). We rejected aerial images with CL edge effects patterns. The presence of more than three diffraction-like patterns of adjacent rays caused rejection of the whole series because we suspected that the lens or the subject had moved. More than four images rejected for any reason caused the rejection of the whole series, which was not used in further processing. This occurred for 17 of a total of 45 series. In exceptional cases (three of 28), we found that the wave aberration corresponding to an apparently normal series of images was very different from the rest of consecutive runs. These abnormal patterns were rarely or never repeated, and we interpreted that they corresponded to unstable positions of the CL or CL shift during the measurement. These abnormal modes usually had also an abnormally high amount of coma and/or astigmatism. All of the aberration estimates presented here were calculated at least from the mean of three series.

\section{RESULTS}

Fig. 1 shows wave aberration maps for all four subjects. For each subject, we show the four measurements performed: total and anterior-surface aberrations with and without CL. Anterior-surface aberrations stand for aberrations of the anterior corneal surface for the eye without CL and for aberrations of the anterior surface of the lens when the eye is wearing the CL. Defocus has been removed in all cases. For each subject, the four upper maps include all aberrations except tilt and defocus, and in the four lower maps, astigmatism has also been removed. To show the effect of CL wear, we have used the same gray scale for anterior-surface and total aberration maps for the same subject. Contours have been plotted at $1-\mu \mathrm{m}$ intervals. Pupil size is $5 \mathrm{~mm}$ in all cases. In many cases, and most obviously for subject $S 1$, the number of contour lines is lower with the CL, indicating a correction of the natural aberrations by the CL. Not only does astigmatism decrease (see upper maps), but also higher-order aberrations. We found an increase of aberrations with CL only for subject S4, whose natural aberrations were very low. Although the amount of aberrations decreases in most cases, the aberration pattern with CL follows a pattern similar to the natural wave aberration. This is indicative of some degree of conformity. There is a strong similarity between total and anteriorsurface wave aberration maps in all subjects and conditions.

Fig. 2 compares Zernike coefficients for two representative subjects (S1 and S3). S1 (Fig. $2 \mathrm{a}$ and b) is the subject with the highest amount of aberrations without CL's and the highest degree of compensation with RGP CL's. S3 (Fig. $2 \mathrm{c}$ and d) has high astigmatism but low high-order aberrations. The ordering and notation of the Zernike coefficients follows the recommendations of the Optical Society of America Standards Committee. ${ }^{33}$

For subject S1, total astigmatism (terms 3 and 5) is well corrected, but there is also a large reduction of third-and higher-order aberrations - see, for example, coefficient $12\left(Z_{4}^{0}\right)$ and $13\left(Z_{4}^{-2}\right)$ in Fig. 2a. The most significant anterior-surface aberration coefficients are also largely reduced, with the exception of spherical aberration $\left(Z_{4}^{0}\right)$ : astigmatism, terms $3\left(Z_{2}^{2}\right)$ and $5\left(Z_{2}^{-2}\right)$, and comatic term $8\left(Z_{2}^{-1}\right)$ in Fig. 2b. There is a good correspondence between total and anterior-surface aberrations (Fig. $2 \mathrm{a}$ and b).

Subject $\mathrm{S} 3$ shows an aberration pattern dominated by astigmatism (almost as high as S1), practically all corneal in origin, as indicated by the great correspondence of total and corneal aberrations (Fig. 2 c and d). Spherical aberration is the predominant high-order aberration of the cornea, but not of the whole eye. In this subject, we found only a small correction of aberrations by the RGP CL.

Fig. 3 summarizes the effect of RGP CL's on total aberrations (root mean square; RMS) for different orders of the Zernike polynomial expansion for all subjects. Fig. 3a shows RMS for all terms, excluding tilt and defocus. The CL significantly corrected part of the ocular aberrations in three of four subjects. RMS decrements ranged from $0.9 \mu \mathrm{m}$ in S1 to $0.2 \mu \mathrm{m}$ in S3. For subject S4, there was a slight increase in RMS $(0.09 \mu \mathrm{m})$. This value is of the order of the RMS variability ( $0.11 \mu \mathrm{m}$ for this subject), and, therefore, it is not statistically significant. This subject had a low amount of aberrations, and internal optics RMS $(0.37 \mu \mathrm{m})$ is comparable to corneal RMS $(0.51 \mu \mathrm{m})$. Anterior-surface aberrations decreased (RMS 0.51 to 0.46 ), however, with CL wear.

Fig. $3 \mathrm{~b}$ shows RMS for all terms of third- and higher-order. These terms account for all aberrations that cannot be corrected with conventional ophthalmic lenses. Fig. 3c shows RMS for Zernike coefficients of third order only, i.e., coma-like aberrations. Fig. 3d shows fifth- and higher-order aberrations. Subject S1's natural optics showed a high amount of aberrations in all orders, and there was a reduction of aberrations in all orders with RGP CL wear. 


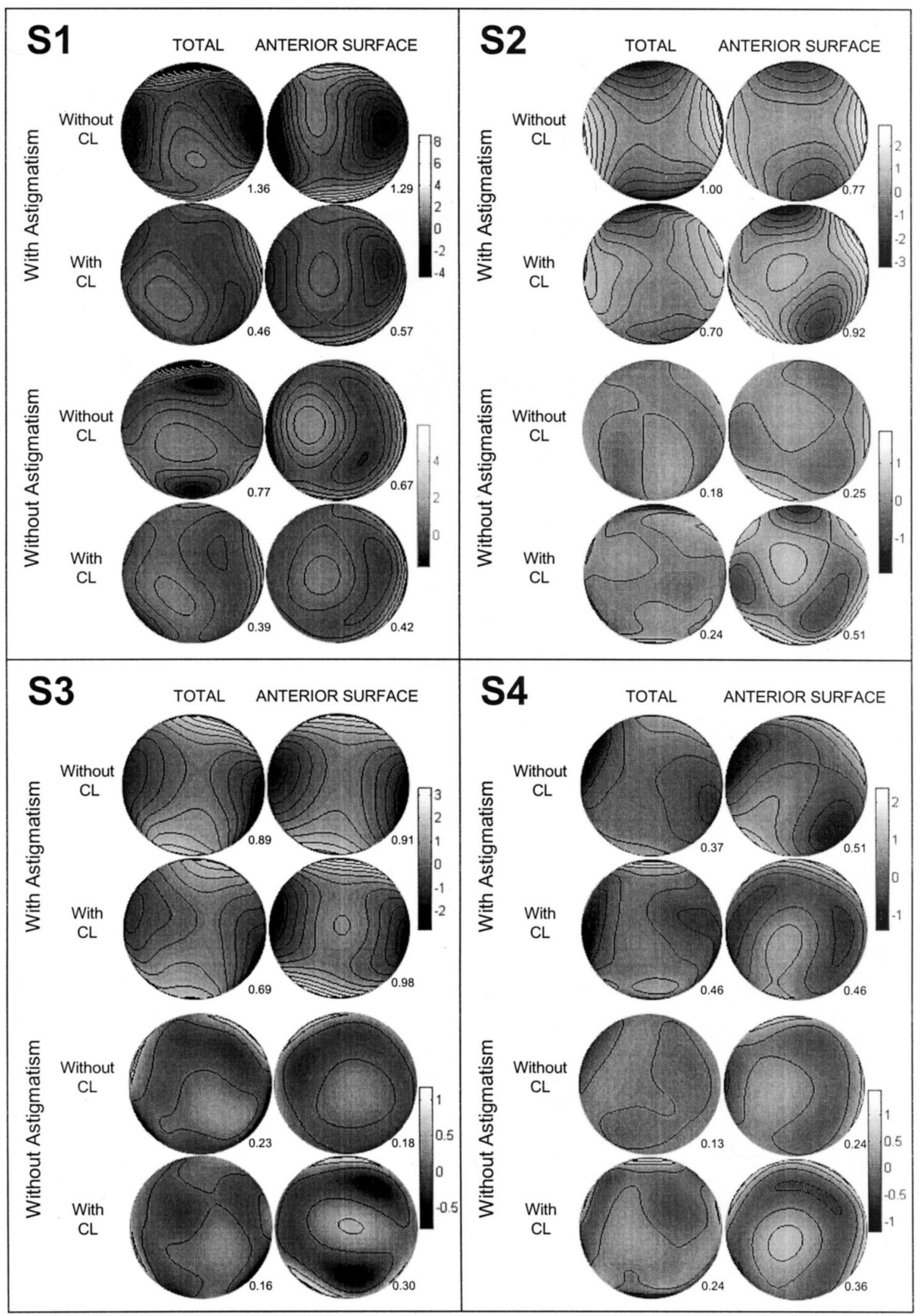

FIGURE 1.

Wave aberration maps for all subjects. For each subject, we show the four wavefronts measured: total (left panels) and anterior surface (right panels), with and without rigid gas permeable contact lens (RGP CL). Anterior-surface aberrations stand for aberrations of the anterior corneal surface for the natural eye, and aberrations of the anterior surface of the CL when the eye is wearing the CL. For each subject, the four upper maps include all aberrations except tilt and defocus, and in the four lower maps, astigmatism has also been removed. Contours have been plotted at a 1- $\mu \mathrm{m}$ interval. Pupil diameter is $5 \mathrm{~mm}$ in all cases. The root mean square (in micrometers) is indicated for each wavefront. 

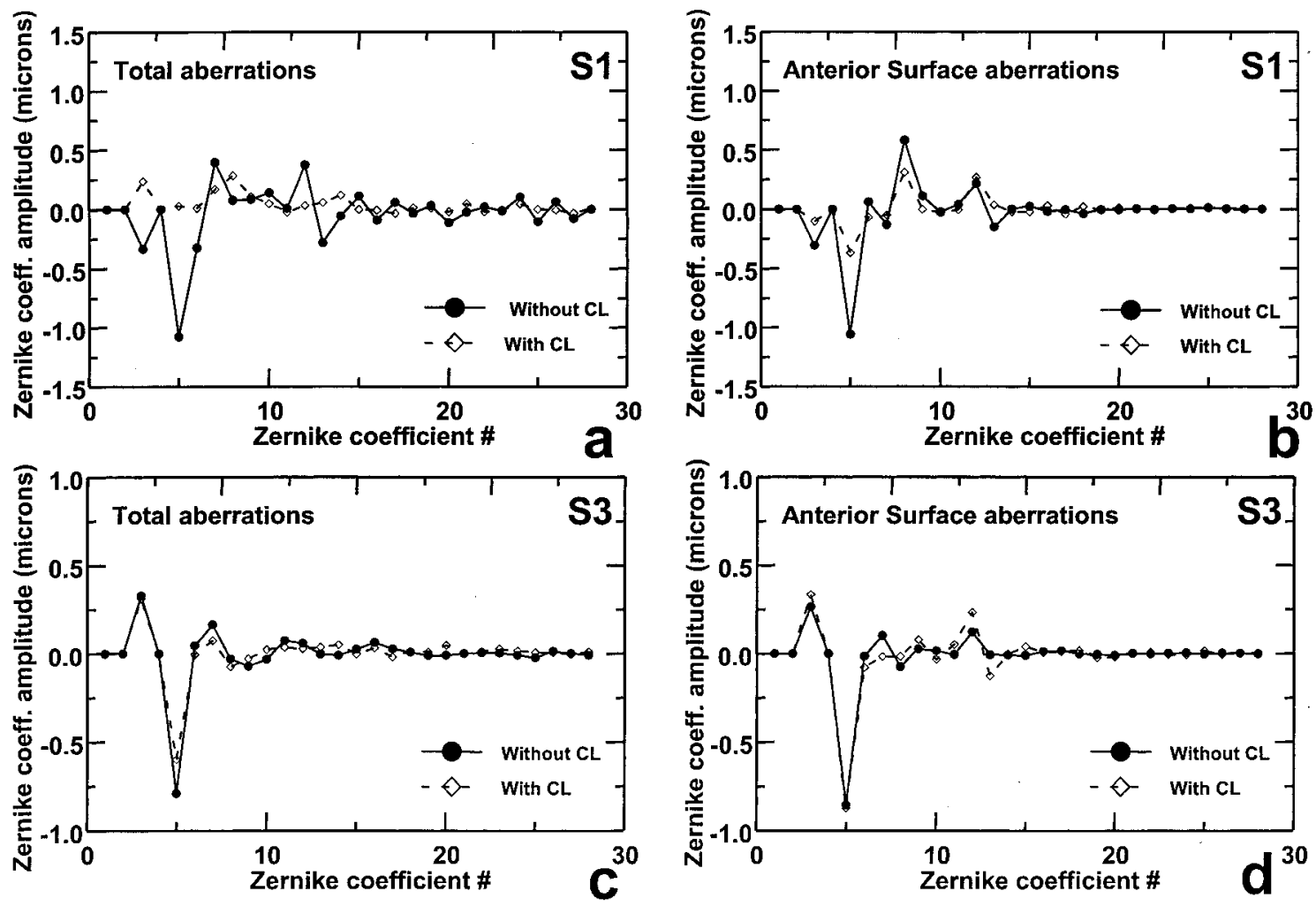

FIGURE 2.

Zernike coefficients for subjects S1 and S3. a: Total aberrations with and without contact lens (CL) for S1; b: anterior-surface aberrations with and without $\mathrm{CL}$ for S1; c: total aberrations with and without CL for S3; d: anterior-surface aberrations with and without CL for S3.
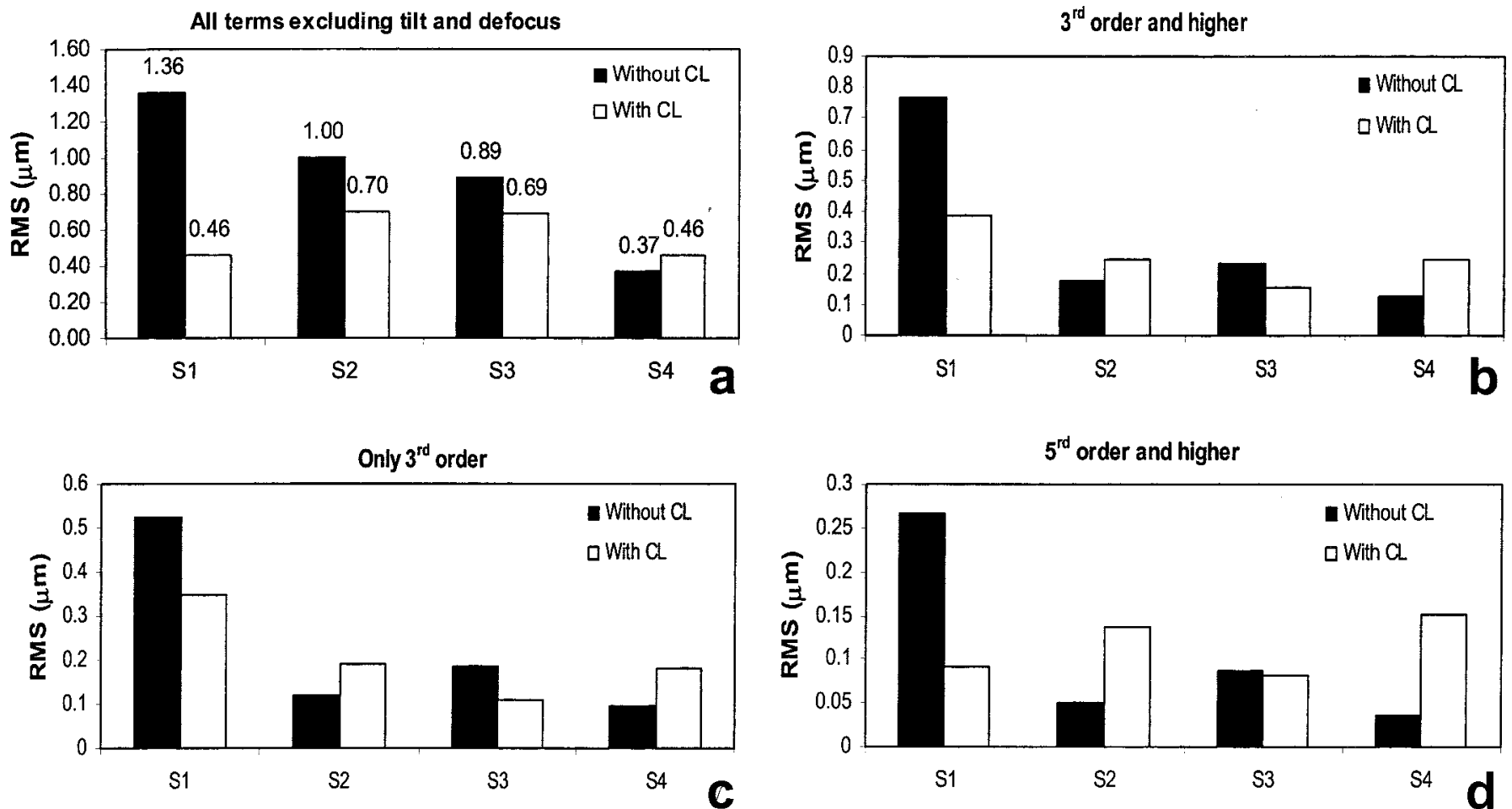

FIGURE 3.

Effect of rigid gas permeable contact lenses $(\mathrm{CL})$ on total root mean square (RMS) for all subjects. a: RMS for all terms, excluding tilt and defocus; b: RMS for third- and higher-order terms; c: RMS for third-order terms; d: RMS for fifth- and higher-order terms. 
All other subjects had a low amount of aberrations without CL, other than astigmatism, and the use of CL's did not change them significantly. The effect of RGP wear on fifth- and higher-order terms (Fig. 3d) showed different trends across subjects: a decrease for S1, no difference for S3, and an increase for S2 and S4. An increase in the fifth-and higher-order terms was curiously found in the same subjects who experienced an increase in third-order terms and more systematic decentrations of the RGP lens. A possible increase of third-and higher-order terms due to decentrations had been predicted, ${ }^{27,34}$ although this effect has proved more relevant for aspheric lenses.

Fig. 4 shows total and anterior-surface fourth-order spherical aberration coefficient $\left(Z_{4}^{0}\right)$ for the different subjects with and without CL. As expected, despite intersubject differences in the amount of spherical aberration in the natural corneas, we obtained the same amount of spherical aberration for all lenses (increased with respect to natural values because the lenses were spherical). However, the total spherical aberration with CL was close to zero for all subjects.

\section{DISCUSSION Aberration Correction}

The principle of correction of corneal irregularities (and hence corneal aberrations) by RGP CL's has been explained by several authors. ${ }^{26,27}$ The CL substitutes the anterior surface of the cornea with a polished regular surface. The main refraction is now produced at the anterior surface of the CL. Tear between lens and cornea fills in corneal irregularities. The power of the anterior surface of the cornea decreases to $11 \%$ because refraction indexes are almost the same $\left(\mathrm{n}_{\text {tear }}=1.336, \mathrm{n}_{\text {cornea }}=1.376\right)$. Therefore, the CL correction acts on corneal aberrations. We have found that RGP CL's are able to correct ocular aberrations to some extent. For the most aberrated eye, we found a decrease of RMS (excluding tilt and defocus) by a factor of three (or RMS decrement of $0.9 \mu \mathrm{m}$ ). The improvement was not only due to astigmatism corrections. Nonconventional aberrations were also significantly reduced: third- and higher-order RMS decreased by a factor of two (a decrement of $0.38 \mu \mathrm{m}$ ). Interestingly, total spherical aberration was close to zero, despite the spherical surfaces of the CL. These results

\section{Spherical Aberration}

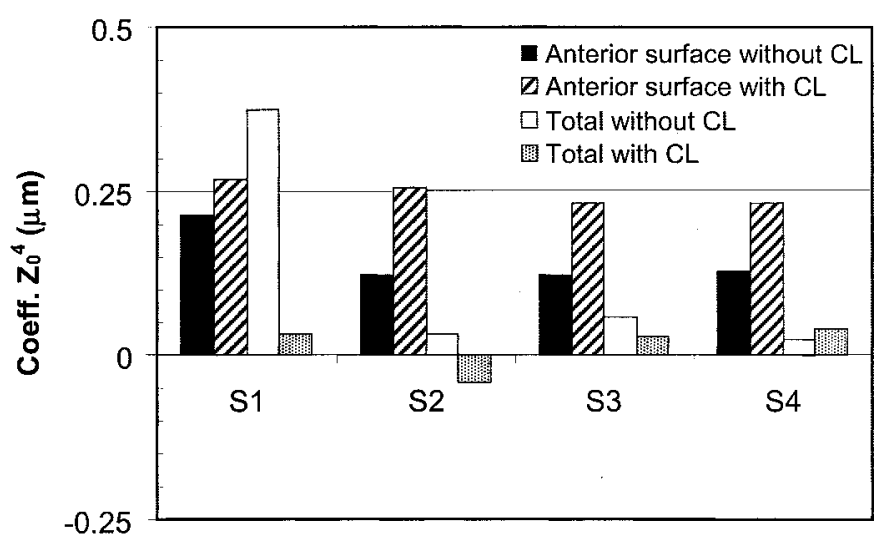

FIGURE 4.

Total and anterior-surface fourth-order spherical aberration coefficient $\left(Z^{0}{ }_{4}\right)$ for the four subjects, with and without contact lenses $(\mathrm{CL})$. are comparable to the best case reported by Hong et al. ${ }^{29}$ (RMS decreasing from 0.5 to $0.14 \mu \mathrm{m}$ ), who compared the impact of RGP CL's, soft CL, and spectacles on ocular aberrations. This degree of compensation is close to that achieved by some custom correction methods, which aim at correcting not only corneal but the whole optical system aberrations. Navarro et al..$^{21}$ reported an increase by a factor of five (RMS decrease from 1.25 to 0.25 for $6.5-\mathrm{mm}$ pupils) using static correction by custom phase plates. Preliminary results with custom CL's showed a decrease in RMS from 0.83 to 0.35 , for 5 -mm pupils. ${ }^{22}$ In addition, the first outcomes of custom refractive surgery showed variable results. ${ }^{23,}{ }^{24} \mathrm{~A}$ decrease of the RMS from 1.5 to $0.2 \mu \mathrm{m}$ for $6.8-\mathrm{mm}$ pupils have been reported using dynamic corrections with adaptive optics. ${ }^{19,20}$ These findings may have some implications in myopia management and control. Several clinical trials ${ }^{35,36}$ have found that children wearing RGP CL had a slower myopia progression than other age- and refraction-matched groups wearing glasses or soft CL's. The differences could not be explained by corneal flattening in RGP CL wearers. Degraded vision with occluding diffusers (and conceivably with an increased amount of aberrations) has been linked to myopia development both in animal models and humans. A better optical quality with RGP CL's may be one of the causes for this apparent slow in myopia progression.

The major limitation of aberration correction with standard RGP CL's is that it is restricted to anterior corneal surface aberrations, whereas the previous methods aim at canceling all aberrations. We have found that the amount of aberration corrected depends on the subject's initial aberrations and, in particular, whether the ocular aberration pattern was dominated by corneal aberration or, rather, the internal aberrations played a significant role. Measuring anterior-surface and total aberration allows, by subtraction, an accounting for the contribution of internal aberrations to the ocular optics. Previous studies have applied these comparisons to study the interaction of the aberrations of the different ocular components as a function of age, ${ }^{7}$ refractive error, ${ }^{37}$ or in refractive surgery. ${ }^{1}$ It appears that in young, normal eyes, there is an important degree of balance between corneal and internal aberrations. The measure of anterior-surface and total aberrations in patients with and without RGP CL's allowed us for the first time to evaluate the interactions of the ocular components (including the internal optics) with RGP CL's and understand the performance of this type of lens individually. From our subjects (Figs. 1 through 3), $\mathrm{S} 1 \mathrm{had}$ the greatest amount of corneal aberrations (RMS = $1.29 \mu \mathrm{m}$, including astigmatism), and the ocular aberration pattern was dominated by corneal aberrations. This is the most favorable case to achieve a good aberration correction with CL's and explains the excellent outcomes for this subject. Subjects S2 and S3 had low internal aberrations (RMS $=0.42 \mu \mathrm{m}$ and $0.3 \mu \mathrm{m}$, respectively) but only moderate corneal aberrations (RMS $=0.77$ $\mu \mathrm{m}$ and $0.91 \mu \mathrm{m}$, respectively), and, therefore, the correction was not so remarkable. Subject $S 4$ had very low corneal aberration $(\mathrm{RMS}=0.51 \mu \mathrm{m})$, which was partially compensated by internal aberrations (RMS $=0.37 \mu \mathrm{m}$ ), producing very low total aberrations (RMS $=0.36 \mu \mathrm{m}$ ). In this case, aberration correction was not achieved despite the fact that anterior-surface aberrations were decreased by the RGP lens (from $0.51 \mu \mathrm{m}$ to $0.46 \mu \mathrm{m}$ ).

Our study demonstrates that internal optics limit the aberration correction by the use of RGP CL's. However, in subjects with 
predominant corneal aberrations, corrections can be of the same order as those achieved by custom devices. This is particularly relevant in cases where increased aberrations limit visual performance, such as pathological or surgical corneas. All previous studies of aberration compensation made use of customized optical elements, subjects with increased aberrations by corneal pathology, or specially manufactured CL's, whereas we studied normal subjects wearing their own standard spherical RPG CL's.

It may be argued that the subjects in our study may not be considered normal because it is well known that long-term RGP lens wear can alter corneal shape and induce corneal warpage and distortion. ${ }^{38}$ If that was the case, the potential benefits of RGP CL's to improve the optical quality may be overestimated. We compared third- and higher-order corneal aberrations in our four subjects with a population of other 38 normal young ( $31 \pm 7$ years) myopes $(-4 \pm 2.2 \mathrm{D})$ who were measured using the same procedures. For this control group, third- and higher-order corneal RMS was $0.57 \pm 0.2 \mu \mathrm{m}$. This value was close to the RMS (0.67 $\mu \mathrm{m})$ for the most aberrated subject $S 1$ in our study, who had been wearing RGP CL's for more than 10 years. Corneal aberrations in the other three subjects were lower than $0.25 \mu \mathrm{m}$, as seen in Fig. 1 . Therefore, the results found in the study are not necessarily unique to this particular set of subjects, and most subjects from the control group could potentially benefit from a reduction of aberrations by RGP CL's.

\section{Flexure}

When placed on the eye, the anterior surface of RGP CL's is not perfectly regular but it is also affected by corneal shape. Depending on some factors (lens thickness, flexibility, etc.) lenses flex to some degree to conform the corneal shape. ${ }^{39}$ Previous studies have used videokeratographic elevation maps to estimate the degree of irregularity introduced by flexure. ${ }^{28}$ To our knowledge, no previous study estimated real optical aberrations from those maps. The impact of flexure on visual performance is not clear. In our study, the four complementary measurements (with and without lens, anterior-surface and total aberration) provide information to assess the impact of flexure not only on anterior-surface but also on total aberrations.

Wave aberration maps with and without CL's in Fig. 1 show some similarities. This is particularly evident for astigmatism. Fewer contour lines are indicative of lower amount of astigmatism, but the angle is generally preserved. To study the degree of conformity between the fitted lens and the underlying cornea in terms of aberrations, we have correlated corneal Zernike coefficients with and without $\mathrm{CL}$ on (Fig. 5). Because fifth- and higher-order aberrations are close to zero, only coefficients up to the fourth order are included. Slopes for each subject's best linear fit and regression coefficients (R) are also shown in Fig. 5. A slope between 0 and 1 is indicative of some degree of compensation by the CL: the lower the slope, the larger the compensation. A regression coefficient close to unity indicates that both anterior-surface wavefronts, with and without lens, show similar shape, i.e., that the lens conforms to the corneal shape. Spherical aberration has not been included in the computation because no correlation was found. This issue will be discussed in detail in the next subsection. We have checked that the inclusion of the spherical term did not substantially change the correlation values. Subject $S 1$ showed the highest correction (slope $=0.37)$ and high conformity $(\mathrm{r}=0.94)$. Subject $\mathrm{S} 2$ showed moderate correction and conformity (slope $=0.92, r=0.76$ ). Subject $\mathrm{S} 3$ showed no correction and very high conformity (slope $=1.02$, $r=0.97$ ), indicating that the lens flexure prevented correction. Subject $\$ 4$ had good corneal correction and low conformity (slope $=0.53, \mathrm{r}=0.65)$.

This analysis agrees well with results shown in Fig. 3a, except for subject S4, for whom there was good corneal correction but an increase in total RMS. This can be explained by the contribution of internal aberrations. Flexure analysis relates solely to anterior-surface aberrations, and for this subject, internal aberrations did partially compensate for corneal aberrations.

Although Fig. 5 analyzes all Zernike coefficients together, the effect of lens flexure can be studied in each term individually. Optimization of aberration compensation may be achieved by using individual total and corneal aberration data and a careful custom control of lens flexure.

Astigmatism was mostly of corneal origin in all subjects except S4. For S1, there was an important decrease in astigmatism $(-1.39$ D) by RGP CL wear. For S2 and S3 there was only a slight decrease in total astigmatism because the lens followed the corneal astigmatism almost completely. It is interesting to describe the effects of lens flexure and the different ocular component contributions to astigmatism for subject S4. For S4, internal and corneal astigmatism had values of $-0.71 \mathrm{D}$ and $-0.47 \mathrm{D}$ but at angles of $142^{\circ}$ and $29^{\circ}$. This indicates that corneal and crystalline lens astigmatism had nearly opposite directions (deviation from complete opposition is $23^{\circ}$ ), so they were partially compensating each other to produce a final astigmatism of -0.54 at $162^{\circ}$ as measured by LRT (or -0.50 at $159^{\circ}$ as measured with the autorefractor). The RGP lens corrected part of the corneal astigmatism, but introduced a slight rotation of $19^{\circ}$ (Fig. 1). The deviation from opposition is now $42^{\circ}$, making the compensation less effective. The final astigmatism slightly increased to $-0.61 \mathrm{D}$. This detailed study can be performed on higher-order aberration terms other than astigmatism.

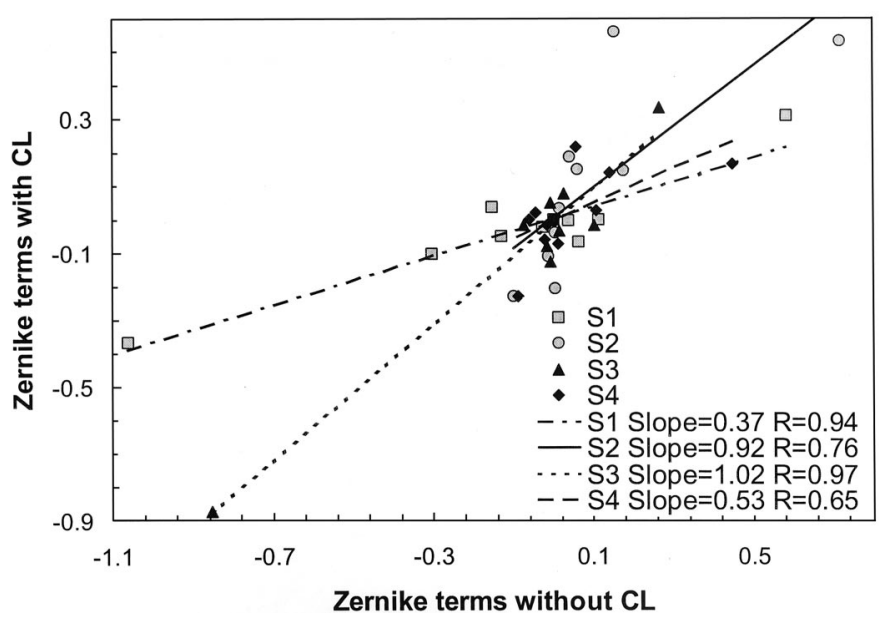

FIGURE 5.

Correlation between anterior-surface Zernike coefficients with and without contact lenses (CL). Only coefficients up to the fourth order are included. Spherical aberration was not included. Slopes for each subject's best linear fit and regression coefficients $(R)$ are also shown. 
This individual analysis of the effects of lens flexure and the contribution of corneal and internal aberrations in each particular eye suggests that a control of the lens flexure can optimize aberration compensation, depending on the particularities of each eye. In eyes dominated by corneal aberrations (such as $S 1$ ), high rigidity is advisable. However, for eyes where corneal and internal aberrations are well balanced (among our subjects, $S 4$ is the closest case) a high conformity to the corneal shape would produce best results.

All previous analysis has been done for aberrations other than spherical aberration. We did not find that spherical aberration was affected by flexure (Fig. 4). Several studies report ${ }^{39}$ that lens flexure affects the amount of measured regular and irregular astigmatism in astigmatic corneas wearing RGP lenses, and this effect depends mostly on lens thickness and, to a lesser extent, lens material. However, no evidence of flexure has ever been found on spherical corneas, suggesting that RGP lenses do not conform to symmetric corneal shapes. ${ }^{39}$

\section{Tear Lens Aberrations}

The spherical aberration of the natural cornea is positive in all eyes with values varying across subjects (Fig. 4). The anterior surface of the CL showed larger amounts of positive spherical aberration, consistent with spherical lenses and very similar across subjects. The total spherical aberration was higher than the corneal aberration in one subject (S1), indicating that the crystalline lens added up to the spherical aberration of the cornea. In the rest of the subjects, we found the previously reported trend that internal negative spherical aberration partially balances the positive corneal spherical aberration, resulting in a low total spherical aberration. ${ }^{40}$ Despite the positive spherical aberration contribution of the RGP CL, as estimated from videokeratographic measurements, we found very low values of total spherical aberration with the RGP CL. Our results suggest that there is a compensation of the positive spherical aberration induced by the CL by the tear lens (i.e., the tear film meniscus between the back surface of the CL and the anterior corneal surface). In the natural eye, the internal aberrations (total minus corneal aberrations) account for the internal ocular optics. In the eye wearing the CL, internal aberrations include the mentioned internal optics and the tear lens. The difference of the internal aberrations from measurements with the $\mathrm{CL}$ and the internal aberrations in natural conditions represents the tear lens aberrations.

Although this is an indirect and somewhat noisy estimate (0.13- $\mu \mathrm{m}$ standard deviation, on average, for the spherical aberration coefficient), because it is computed after a double subtraction, we found a common trend in all the tear lens aberration maps from our four subjects. The spherical aberration is typically the largest high-order coefficient, and it is always negative (S1: $-0.39 \mu \mathrm{m}$; S2: $-0.21 \mu \mathrm{m}$; $33:-0.14 \mu \mathrm{m}$; $S 4:-0.09 \mu \mathrm{m}$ ), which explains the described compensation of the lens positive anterior spherical aberrations.

A potential compensation by the tear lens of other aberrations, such as astigmatism, has been suggested before. ${ }^{25}$ As an example, Fig. 6 shows the tear lens wave aberration map for S2 (right panel), estimated as the difference of internal aberrations with CL (left panels) and without CL (central panels). Negative spherical aberration dominated the wave aberration tear lens aberration pattern.

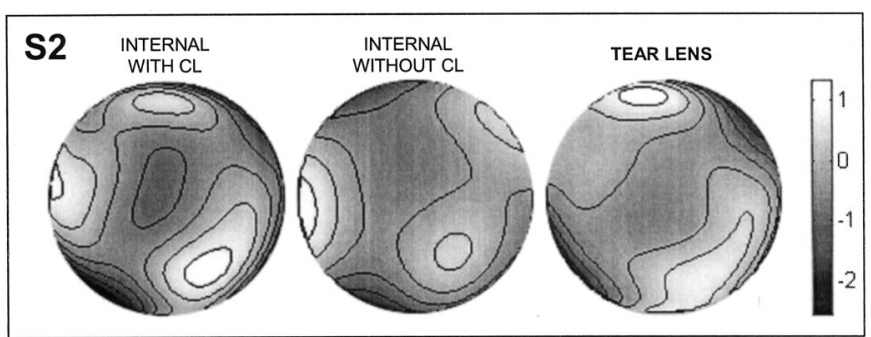

\section{FIGURE 6.}

Tear lens wave aberration map for subject S2 (right panel), estimated as the difference of internal aberrations with contact lenses (CL) (left panel) and without $\mathrm{CL}$ (central panel).

We performed a computer simulation to assess potential sources for this additional negative spherical aberration. Using ray tracing, we computed the spherical aberration due only to the tear lens. ${ }^{34}$ We assumed different asphericities for the posterior CL surface (which acted as the anterior surface of the tear lens). We used the actual subjects' anterior corneal surfaces as the posterior surface of the tear lens. We calculated that for spherical posterior lens surfaces, the tear lens spherical aberration was negative in all cases, following the trend found experimentally. However the estimated amount $(-0.016 \pm 0.002$ on average across subjects) was much lower. The spherical aberration of the tear lens depends on the asphericity of the posterior lens surface. We found that an increase in the asphericity shifted the spherical aberration of the tear lens toward more negative values (for example, the mean spherical aberration for asphericity 0.6 was $-0.063 \pm 0.004)$. Nominal asphericities of the posterior surface of the lens are zero (spherical surfaces) for all subjects except for subject S3 (see Table 1). However, it is well known that nominal specifications of RGP lenses can vary from actual values, particularly for the posterior surface. ${ }^{41}$ Deviations from ideal spheres may not be unlikely and may be a potential cause for the increased (in this case beneficial) negative spherical aberration.

\section{CONCLUSIONS}

In this study, we have shown the application of combined measurements of aberrometry and corneal topography in RPG CL fitting. By using this methodology, we have been able to evaluate the contribution of the anterior surface of the RGP CL, the internal ocular optics, the flexure, and the tear lens aberrations on the optical performance of eyes wearing RGP CL. This information provides an accurate analysis of CL fitting in individual eyes, and allows, for example, for tracking of individual aberration terms through the different optical elements involved (contact lens, tear lens, cornea, and internal optics).

We have shown that RGP CL's can significantly improve the natural optics of the subject, provided that corneal aberrations are predominant and the lens flexure is well controlled. Our study concludes that the lens flexure (or conformity) has a great impact on the degree of corneal aberration compensation. Because there is previous evidence that internal aberrations usually compensate to some extent the aberrations of the cornea, ${ }^{40}$ we conclude that a custom control on lens flexure can improve the cornea/internal 
optics aberration ratio and result in an improvement of the subject's visual performance.

Finally, we have shown that spherical RGP CL's do not induce higher final spherical aberration on the eyes measured. Although the anterior lens surface shows higher spherical aberration than the natural cornea, we found negligible values of total spherical aberration in all four subjects wearing their RGP CL's. Our results suggest that this compensation is produced by the tear lens, but this could be confirmed using measurements on a larger population and a strict verification of the parameters of the lens used.

\section{ACKNOWLEDGMENTS}

Funded by grant CAM 08.7/0010/2000 (Comunidad Autónoma de Madrid, Spain) and BFM 2002-02638 (Ministerio de Ciencia y Tecnologia, Spain) to S. Marcos. C. Dorronsoro thanks CSIC and Alcon Cusi, Spain, for a research fellowship. S. Barbero thanks CSIC, Carl Zeiss, Spain, and Ministerio de Educacion y Cultura for research fellowships. Carl Zeiss, Spain lent a Mastervue Atlas Corneal Topography System. The authors acknowledge Arthur Ho and an anonymous reviewer for helpful suggestions and the four volunteers in the study for their generous participation.

Received May 1, 2002; revision received September 24, 2002.

\section{REFERENCES}

1. Marcos S, Barbero S, Llorente L, Merayo-Lloves J. Optical response to LASIK surgery for myopia from total and corneal aberration measurements. Invest Ophthalmol Vis Sci 2001;42:3349-56.

2. Moreno-Barriuso E, Lloves JM, Marcos S, Navarro R, Llorente L, Barbero S. Ocular aberrations before and after myopic corneal refractive surgery: LASIK-induced changes measured with laser ray tracing. Invest Ophthalmol Vis Sci 2001;42:1396-403.

3. Munson K, Hong X, Thibos LN. Use of a Shack-Hartmann aberrometer to assess the optical outcome of corneal transplantation in a keratoconic eye. Optom Vis Sci 2001;78:866-71.

4. Barbero S, Marcos S, Llorente L. Optical changes in corneal and internal optics with cataract surgery (abstract 388). 2002 Annual Meeting Abstract and Program Planner Association for Research in Vision and Ophthalmology 2002. Available at: www.arvo.org. Accessed September 30, 2002.

5. Artal P, Marcos S, Navarro R, Miranda I, Ferro M. Through-focus image quality of eyes implanted with monofocal and multifocal intraocular lenses. Optical Engineering 1995;34:772-9.

6. McLellan JS, Marcos S, Burns SA. Age-related changes in monochromatic wave aberrations of the human eye. Invest Ophthalmol Vis Sci 2001;42:1390-5.

7. Artal P, Berrio E, Guirao A, Piers P. Contribution of the cornea and internal surfaces to the change of ocular aberrations with age. J Opt Soc Am (A) 2002;19:137-43.

8. He JC, Burns SA, Marcos S. Monochromatic aberrations in the accommodated human eye. Vision Res 2000;40:41-8.

9. Barbero S, Marcos S, Merayo-Lloves J, Moreno-Barriuso E. Validation of the estimation of corneal aberrations from videokeratography in keratoconus. J Refract Surg 2002;18:263-70.

10. Collins MJ, Wildsoet CF, Atchison DA. Monochromatic aberrations and myopia. Vision Res 1995;35:1157-63.

11. Marcos S, Moreno-Barriuso E, Llorente L, Navarro R, Barbero S. Do myopic eyes suffer from larger amount of aberrations? In: Thorn F, Troilo D, Gwiazda J, eds. Myopia 2000. Proceedings of the 8th International Conference on Myopia. Boston: International Conference on Myopia 2000 Press, 2000:118-21.

12. Moreno-Barriuso E, Marcos S, Navarro R, Burns SA. Comparing laser ray tracing, the spatially resolved refractometer, and the Hartmann-Shack sensor to measure the ocular wave aberration. Optom Vis Sci 2001;78:152-6.

13. Moreno-Barriuso E, Navarro R. Laser ray tracing versus HartmannShack sensor for measuring optical aberrations in the human eye. J Opt Soc Am (A) 2000;17:974-85.

14. Schultze RL. Accuracy of corneal elevation with four corneal topography systems. J Refract Surg 1998;14:100-4.

15. Schwiegerling J, Greivenkamp JE. Using corneal height maps and polynomial decomposition to determine corneal aberrations. Optom Vis Sci 1997;74:906-16.

16. Applegate RA, Hilmantel G, Howland HC, Tu EY, Starck T, Zayac EJ. Corneal first surface optical aberrations and visual performance. J Refract Surg 2000;16:507-14.

17. Barbero S, Marcos S, Merayo-Lloves JM. Corneal and total optical aberrations in an unilateral aphakic patient. J Cataract Refract Surg 2002;28:1594-600.

18. Guirao A, Artal P. Corneal wave aberration from videokeratography: accuracy and limitations of the procedure. J Opt Soc Am (A) 2000; 17:955-65.

19. Fernandez EJ, Iglesias I, Artal P. Closed-loop adaptive optics in the human eye. Opt Lett 2001;26:746-8.

20. Hofer H, Chen L, Yoon GY, Singer B, Yamauchi Y, Williams DR. Improvement in retinal image quality with dynamic correction of the eye's aberrations. Optics Express 2001;8:631-43. Available at: http:// www.opticsexpress.org/oearchive/source/31887.htm. Accessed September 30, 2002.

21. Navarro R, Moreno-Barriuso E, Bará S, Mancebo T. Phase-plates for wave-aberration compensation in the human eye. Opt Lett 2000;25: 236-8.

22. Lopez-Gil N, Benito A, Castejón-Mochón JF, Marín JM, Lo-a-Foe G, Marin G, Germigier B, Joyeux D, Chateau N. Artal P. Aberration correction using customized soft contact lenses with aspheric and asymmetric surfaces (presentation 973). 2002 Annual Meeting Abstract and Program Planner Association for Research in Vision and Ophthalmology 2002. Available at: www.arvo.org. Accessed September 30, 2002.

23. MacRae SM, Schwiegerling J, Snyder R. Customized corneal ablation and super vision. J Refract Surg 2000;16:S230-5.

24. Mrochen M, Kaemmerer M, Seiler T. Wavefront-guided laser in situ keratomileusis: early results in three eyes. J Refract Surg 2000;16: 116-21.

25. Phillips AJ, Speedwell L. Contact Lenses, 4th ed. Oxford: Butterworth-Heinemann, 1997.

26. Griffiths M, Zahner K, Collins M, Carney L. Masking of irregular corneal topography with contact lenses. CLAO J 1998;24:76-81.

27. Atchison DA. Aberrations associated with rigid contact lenses. J Opt Soc Am (A) 1995;12:2267-73.

28. Collins MJ, Franklin R, Carney LG, Bergiel C, Lagos P, Chebib D. Flexure of thin rigid contact lenses. 2001;24:59-64.

29. Hong X, Himebaugh N, Thibos LN. On-eye evaluation of optical performance of rigid and soft contact lenses. Optom Vis Sci 2001;78: 872-80.

30. Navarro R, Losada MA. Aberrations and relative efficiency of light pencils in the living human eye. Optom Vis Sci 1997;74:540-7. (Published erratum appears in Optom Vis Sci 1997;74:1049.)

31. Navarro R, Moreno-Barriuso E. Laser ray-tracing method for optical testing. Opt Lett 1999;24:951-3.

32. Llorente L, Diaz-Santana L, Lara-Saucedo D, Marcos S. Aberrations of the human eye in visible and near infrared illumination. Optom Vis Sci 2003:80:26-35.

33. Thibos LN, Applegate RA, Schwiegerling JT, Webb R. Standards for reporting the optical aberrations of eyes. In: Lakshminarayanan $\mathrm{V}$, ed. 
Trends in Optics and Photonics. Vision Science and Its Applications, Vol 35. OSA Technical Digest Series Washington, DC: Optical Society of America, 2000:232-44.

34. Ho A, Manns F, Pham T, Farrar S. The Effect of RGP lens decentration on aberration: the influence of the tear lens [presentation 3105]. 2002 Annual Meeting Abstract and Program Planner Association for Research in Vision and Ophthalmology 2002. Available at: www. arvo.org. Accessed September 30, 2002.

35. Perrigin J, Perrigin D, Quintero S, Grosvenor T. Silicone-acrylate contact lenses for myopia control: 3-year results. Optom Vis Sci 1990;67:764-9.

36. Khoo CY, Chong J, Rajan U. A 3-year study on the effect of RGP contact lenses on myopic children. Singapore Med J 1999;40:230-7.

37. Marcos S, Barbero S, Llorente L. Why high myopic eyes tend to be more aberrated? Optical Society of America Annual Meeting, Long Beach, CA, 2001. Washington, DC: Optical Society of America, 2001:75.
38. Novo AG, Pavlopoulos G, Feldman ST. Corneal topographic changes after refitting polymethylmethacrylate contact lens wearers into rigid gas permeable materials. CLAO J 1995;21:47-51.

39. Corzine JC, Klein SA. Factors determining rigid contact lens flexure. Optom Vis Sci 1997;74:639-45.

40. Artal P, Guirao A. Contributions of the cornea and the lens to the aberrations of the human eye. Opt Lett 1998;23:1713-5.

41. Defazio AJ, Lowther GE. Inspection of back surface aspheric contact lenses. Am J Optom Physiol Opt 1979;56:471-9.

Carlos Dorronsoro

Instituto de Óptica "Daza de Valdés" Consejo Superior de Investigaciones Cientificas

Serrano 121

28006 Madrid

Spain

e-mail:carlos@dorronsoro.com 